\title{
Effects of olanzapine on cytokine profile and brain-derived neurotrophic factor in drug-naive subjects with first-episode psychosis
}

\author{
ERIFILI HATZIAGELAKI ${ }^{1}$, ANASTASIA TSIAVOU ${ }^{1}$, CHARILAOS GERASIMOU $^{2}$, GEORGE D. VAVOUGIOS ${ }^{3}$, \\ ARIS SPATHIS $^{1}$, EFSTATHIOS LASKOS ${ }^{4}$, CHARALABOS PAPAGEORGIOU $^{5}$, ATHANASIOS DOUZENIS $^{2}$, \\ NIKOS CHRISTODOULOU ${ }^{6}$, NICOLAOS STEFANIS ${ }^{5}$, DEMETRIOS A. SPANDIDOS ${ }^{7}$, \\ NIKOLAOS NIKOLAKAKIS ${ }^{8}$, KONSTANTINOS TSAMAKIS ${ }^{2}$ and EMMANOUIL RIZOS ${ }^{2}$ \\ ${ }^{1}$ Second Department of Internal Medicine-Propaedeutic, Research Institute and Diabetes Centre, and \\ ${ }^{2}$ Second Department of Psychiatry, University General Hospital 'ATTIKON', School of Medicine, \\ National and Kapodistrian University of Athens, 12462 Athens; ${ }^{3}$ Department of Respiratory Medicine, \\ University of Thessaly, School of Medicine, Biopolis, 41100 Larissa; ${ }^{4}$ Department of Biochemistry and Microbiology, \\ Athens Psychiatric Hospital 'Dromokaition', 12461 Athens; ${ }^{5}$ First Department of Psychiatry, \\ Eginition Psychiatric Hospital, School of Medicine, National and Kapodistrian University of Athens, \\ 11528 Athens, Greece; ${ }^{6}$ Department of Psychological Medicine, Nottinghamshire Healthcare NHS Trust, \\ University of Nottingham Medical School, Nottingham NG7 2RD, UK; 7 Laboratory of Clinical Virology, Medical School, \\ ${ }^{8}$ Department of Psychiatry and Behavioral Sciences, Faculty of Medicine, University of Crete, 71003 Heraklion, Greece
}

Received January 3, 2019; Accepted February 14, 2019

DOI: $10.3892 /$ etm.2019.7285

\begin{abstract}
Immunological abnormalities have been implicated in schizophrenia. On the other hand, antipsychotics may exert immunomodulatory effects, by triggering pro-inflammatory and anti-inflammatory agents through complex homeostatic mechanisms, which seem to be implicated in medication responsiveness and in the presence or not of adverse effects. There is evidence that olanzapine, a second generation antipsychotic, may increase synapse formation and neurogenesis through alterations in the levels of cytokines and neurotrophic factors. In the present study, we recruited 14 drug-naive inpatients with first-episode schizophrenia (male:female ratio, 7:7) with a mean age of 26.5 years. The positive and negative syndrome scale (PANSS) scores and serum levels of a broad spectrum of cytokines and of brain-derived neurotrophic factor (BDNF) were recorded twice, once at baseline prior to the initiation of olanzapine treatment and 8 weeks later, once the dose of olanzapine had stabilized. Subsequently, the associations between the PANSS scores and the measured markers were examined. Correlation analyses revealed
\end{abstract}

Correspondence to: Dr Emmanouil Rizos, Second Department of Psychiatry, University General Hospital 'ATTIKON', School of Medicine, National and Kapodistrian University of Athens, Rimini 1, Chaidari, 12462 Athens, Greece

E-mail: erizos@med.uoa.gr

Key words: cytokines, interleukin-6, interleukin-27, brain-derived neurotrophic factor, first-episode, psychosis, olanzapine

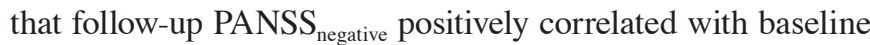
interleukin (IL)-6 $(\mathrm{Q}=0.685, \mathrm{P}=0.007)$ and baseline IL-27 levels $(\mathrm{Q}=0.785, \mathrm{P}=0.001)$. Furthermore, the percentage change in $\mathrm{PANSS}_{\text {negative }}\left[\left(\mathrm{PANSS}_{-}\right.\right.$follow-up $\left.\mathrm{PANSS}_{\text {}_{\text {baseline }}}\right) / \mathrm{PANSS}$-baseline; $\left.\left.\triangle \mathrm{PANSS}_{\text {negative\%}}\right)\right]$ positively correlated with baseline IL-27 $(\varrho=0.785, \mathrm{P}=0.001)$ and baseline IL-6 levels $(\varrho=0.685, \mathrm{P}=0.007)$. Finally, linear regression revealed that follow-up $\mathrm{PANSS}_{\text {negative }}$ was associated with baseline IL-27 $\left(\mathrm{R}^{2}=0.301, \mathrm{P}=0.042\right)$, $\triangle$ PANSS $_{\text {negative\% }}$ was associated with baseline IL-6 $\left(\mathrm{R}^{2}=0.301\right.$, $\mathrm{P}=0.042)$ and baseline IL-27 levels $\left(\mathrm{R}^{2}=0.446, \mathrm{P}=0.009\right)$. Thus, these findings indicate that IL-27 and IL- 6 may be trait markers in patients being administered olanzapine monotherapy at the onset of schizophrenia. However, further studies are warranted in order to replicate these associations and to confirm their potential use as biomarkers of treatment effectiveness and safety, as well as to explore novel immunomodulatory strategies for the treatment of schizophrenia.

\section{Introduction}

Schizophrenia is a severe and highly disabling mental illness, with a complex and heterogeneous etiology, with lifetime prevalence ranging from $0.3-0.66$ to $2.3 \%$, depending on the diagnostic criteria (1). There are numerous theories on the causality of schizophrenia, involving developmental or neurodegenerative processes, neurotransmitter abnormalities and infectious or autoimmune processes. Environmental factors linked to schizophrenia include prenatal infection, obstetric complications, brain injury, cannabis use and birth in winter (2). Patients with schizophrenia experience higher morbidity from infectious and autoimmune diseases, suggesting a 
possible causal link between inflammatory processes and schizophrenia. This is corroborated by consistent reports of abnormalities in immune-inflammatory pathways in schizophrenia (3).

Evidence for immune system abnormalities in schizophrenia includes microglial activation in the brain $(4,5)$ and alterations in cytokine levels in the cerebrospinal fluid and blood (6,7). These results suggest that the immune system may be a target for medications in the near future. Second generation antipsychotics and specifically olanzapine (OLZ) seem to exert neurotrophic effects and to promote neuroplasticity and synapse formation through the activation of neurotrophins, as compared to first generation antipsychotics (8).

Several studies have suggested that some antipsychotics exert anti-inflammatory effects and/or pro-inflammatory effects, which may explain response variability and the variable emergence of adverse effects, such as metabolic syndrome, motor adverse effects, as well as others (9). There is evidence that OLZ reduces the levels of pro-inflammatory cytokines, such as interleukin (IL)-1 $\beta$, IL-2, IL-6 soluble IL-6 receptor (sIL-6R) and tumor necrosis factor (TNF)- $\alpha(10,11)$ while it increases the levels of anti-inflammatory cytokines, such as IL-10, and brain-derived neurotrophic factor (BDNF). Through these effects, it may promote neurogenesis and remodeling in the brain, which may in turn confer clinical efficacy (12).

In the present exploratory study, we measured the serum levels of a broad spectrum of pro-inflammatory and anti-inflammatory cytokines, as well as BDNF in 14 drug-naive patients with first-episode psychosis (FEP), first at baseline prior to the initiation of any antipsychotic medication and at 8 weeks later, after achieving the stable dosing of OLZ monotherapy. Clinical psychopathology was assessed using the positive and negative syndrome scale (PANSS), before and after treatment. We subsequently explored the associations between the PANSS scores and the measured biomarkers.

The aim of this study was to explore the effects of OLZ monotherapy on pro- and anti-inflammatory markers in patients with FEP, and to determine the mechanisms through which these immune markers are associated with psychopathological parameters. Ultimately, the hypothesis of this study is that these biomarkers could be proposed as trait markers of OLZ monotherapy in previously drug-naive patients with schizophrenia in their first psychotic episode.

\section{Materials and methods}

Study population. This study included a total of 14 antipsychotic-naïve FEP inpatients admitted to the Psychiatric Department of 'ATTIKON' General Hospital, Athens, Greece. All patients were diagnosed with the following disorders, according to the Diagnostic and Statistical Manual of Mental Disorders, Fourth Edition (DSM-IV) (13): Schizophrenia, schizophreniform disorder, brief psychotic disorder and psychotic disorders not otherwise specified. The sample consisted of 14 individuals ( 7 males and 7 females), between 18 and 37 years of age (mean age, 26.5 \pm 6.02 years), who had not previously been treated with any antipsychotic agent. General exclusion criteria included the following: Acute and chronic general medical conditions linked to abnormalities in immune and/or inflammatory responses, such as infections, HIV, allergies, pregnancy, rheumatic or endocrine diseases.

All participants received OLZ, per os, as standard treatment, under 8 weeks of medication. Blood samples of $10 \mathrm{ml}$ were obtained from all participants upon admission, prior to OLZ initiation, and after 8 weeks of treatment. The dose of OLZ was either $15 \mathrm{mg}(\mathrm{n}=6)$ or $20 \mathrm{mg}(\mathrm{n}=8)$. Benzodiazepines were used PRN in the period between both assessments, with intention to treat anxiety, sleep disturbances and agitation.

This study acquired ethics approval by the University General Hospital 'ATTIKON'. All study participants supplied written informed consent. Participation in the study was voluntary and participants could withdraw at any point without detriment to their care. All participants had their capacity to consent tested by a clinical psychiatrist, while relatives or carers were present to ensure consent was properly informed.

Measurement of serum cytokine levels. Fasting blood was collected from each individual and serum was separated by centrifugation at $2,253 \mathrm{xg}$ for $10 \mathrm{~min}$ and stored at $-20^{\circ} \mathrm{C}$, until further biological measurements were performed. A total of $25 \mu \mathrm{l}$ of serum sample was processed with a multiplex bead based assay (Milliplex kits; Merck-MilliPore, Billerica, MA, USA), according to the manufacturer's instructions, in order to determine the levels of 15 cytokines [IL-2, IL-17F, IL-17A, IL-22, IL-1 $\beta$, IL-21, IL-23, IL-27, IL-4, IL-6, interferon (IFN)- $\gamma$, TNF- $\alpha$ and transforming growth factor (TGF)- $\beta 1$, TGF- $\beta 2$, TGF- $\beta 3$ ]. Measurements and analyses were performed on a Luminex 200 platform (Luminex Corp., Austin, TX, USA). Evaluations were performed at weeks 0 and 8 of antipsychotic treatment.

\section{Measurement of serum BDNF levels}

Preparation of serum and storage. Human sera were collected using Vacutainer tubes (Becton-Dickinson, Rutherford, NJ, USA). Samples were allowed to clot for $30 \mathrm{~min}$ prior centrifugation at $2,383 \mathrm{x} \mathrm{g}$ for $15 \mathrm{~min}$ at $15^{\circ} \mathrm{C}$. Serum was separated and stored at $-20^{\circ} \mathrm{C}$ until analysis.

Measurement of BDNF levels. The BDNF levels were measured from the re-thawed serum samples by using the Quantikine Immunoassay kit (cat. no. DBD000; R\&D Systems, Minneapolis, MN, USA). This was a double antibody sandwich ELISA method. The manufacturer's instructions were followed to develop the kit, to calibrate the method and to measure the samples. The absorbance was measured at $450 \mathrm{~nm}$ and corrected at $570 \mathrm{~nm}$ using a Mediators $\mathrm{PhL}$ microplate reader (Mediator Diagnostika Gmbh, Vienna, Austria). Evaluations were performed at weeks 0 and 8 the antipsychotic treatment.

Clinical measurements. Symptoms of schizophrenia were assessed at baseline and 8 weeks following OLZ treatment for all patients using the PANSS scoring system (14). The PANSS is a medical scale used for the evaluation of the severity of both positive and negative symptoms of schizophrenia and it is considered the most reliable scale for the assessment of antipsychotic treatment efficacy (15). The PANSS was administered by the same rater (E.R.) throughout the study.

Statistical analysis. Data are presented as the means \pm SD and median (IQR) where applicable. Data normality was assessed 
Table I. Demographic and clinical characteristics of the study participants.

\begin{tabular}{lcc}
\hline Patients (n=14) & $\begin{array}{c}\text { Prior to } \\
\text { treatment }\end{array}$ & $\begin{array}{c}\text { After } 8 \text { weeks } \\
\text { of treatment }\end{array}$ \\
\hline $\begin{array}{l}\text { Age (years) } \\
\text { Sex (male/female) }\end{array}$ & $\begin{array}{c}26.50 \pm 6.02 \\
\text { Marital status } \\
\text { (married/unmarried) }\end{array}$ & $7 / 7$ \\
$\begin{array}{l}\text { Education (from primary } \\
\text { to higher; years) }\end{array}$ & $14.50 \pm 1.95$ & \\
$\begin{array}{l}\text { Professional status } \\
\text { (employed/unemployed) }\end{array}$ & $6 / 8$ & \\
$\begin{array}{l}\text { PANSS-positive } \\
\text { PANSS-negative }\end{array}$ & $40.28 \pm 5.19$ & $25.92 \pm 5.35$ \\
\hline
\end{tabular}

PANSS, positive and negative syndrome scale.

Table II. BDNF and cytokine levels at baseline and at 8 weeks of follow-up, following treatment initiation with olanzapine.

\begin{tabular}{lccc}
\hline Measurement & Baseline & Follow-up & P-value \\
\hline BDNF & $12.98(57.74)$ & $17.18(45.73)$ & 0.778 \\
IFN- $\gamma$ & $2.74(195.53)$ & $0.30(306.64)$ & 0.975 \\
IL-1 $\beta$ & $5.13(7.05)$ & $4.68(4.14)$ & 0.258 \\
IL-2 & $4.49(3.62)$ & $4.28(5.32)$ & 0.802 \\
IL-4 & $0.06(0.53)$ & $0.08(0.77)$ & 0.387 \\
IL-6 & $2.85(2.01)$ & $2.57(13.71)$ & 0.140 \\
IL-17A & $10.26(84.59)$ & $7.90(116.27)$ & 0.272 \\
IL-17F & $0.02(0.50)$ & $0.02(0.60)$ & 0.380 \\
IL-21 & $4.28(5.32)$ & $4.28(4.50)$ & 0.844 \\
IL-22 & $0.12(0.23)$ & $0.10(0.28)$ & 0.249 \\
IL-23 & $0.27(2.51)$ & $0.25(3.86)$ & 0.753 \\
IL-27 & $0.67(1.51)$ & $0.59(1.35)$ & 0.777 \\
TNF- $\alpha$ & $18.19(19.77)$ & $17.44(23.76)$ & 0.925 \\
TGF- $\beta 1$ & $41.85(61.17)$ & $34.31(46.82)$ & 0.158 \\
TGF- $\beta 2$ & $0.91(2.74)$ & $1.25(2.69)$ & 0.272 \\
TGF- $\beta 33^{a}$ & & & \\
\hline
\end{tabular}

Measurements are in $\mathrm{ng} / \mathrm{ml}$; median (interquartile range) are reported; P-values reported refer to the related samples Wilcoxon signed rank test. ${ }^{a}$ TGF- $\beta 3$ measurements were performed as intended; however, they were below the assay's detection range and as such, they are not reported or included in the analyses. BDNF, brain-derived neurotrophic factor; IFN- $\gamma$, interferon- $\gamma$; IL, interleukin; TGF, transforming growth factor.

with the Shapiro-Wilk test. Comparisons between measurements performed at different time-points and expressed as continuous variables were performed via the related samples Wilcoxon Signed Ranked Test. Correlations between variables were examined by Spearman's rank (@) correlation coefficient. Associations between continuous variables were assessed via

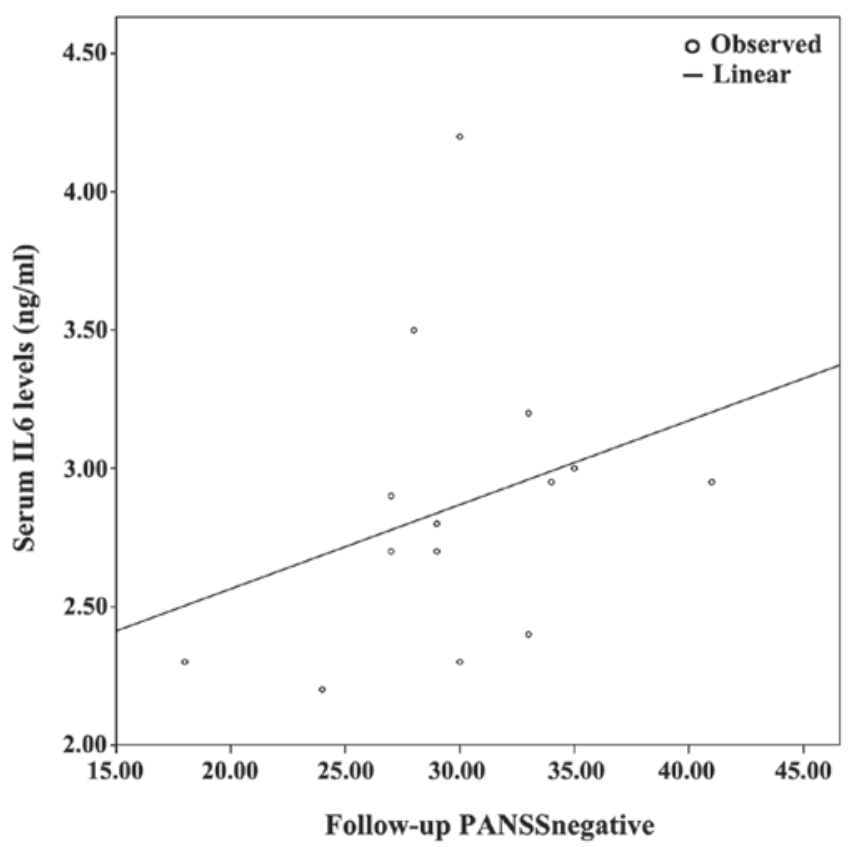

Figure 1. Combined graph depicting the scatterplot and linear regression line between serum IL-6 levels and follow-up PANSS-negative. IL-6, interleukin-6; PANSS, positive and negative syndrome scale.

a linear regression function as determined by the SPSS Curve Estimation module. For all tests, a P-value $<0.05$ was considered to indicate a statistically significant difference.

\section{Results}

The demographics of the study population are presented in Table I, along with an analysis of PANSS subscales between baseline and follow-up. Since at least one of either baseline or follow-up measurements displayed non-parametric distribution based on the Shapiro-Wilk test, non-parametric tests (i.e., related samples Wilcoxon signed rank test and Spearman's @ correlation coeffiecient) were used. Table II presents the cytokine/BDNF levels at baseline and at 8 weeks of follow-up; no statistically significant differences between baseline vs. follow up measurements were detected (related samples Wicloxon signed rank test). Correlation analyses revealed several statistically significant correlations. Specifically, follow-up PANSS negative positively correlated with baseline IL-6 $(\mathrm{Q}=0.685, \mathrm{P}=0.007)$ and baseline IL-27 levels $(\mathrm{Q}=0.785, \mathrm{P}=0.001)$. Furthermore, the percentage change in $\mathrm{PANSS}_{\text {negative }}\left[\mathrm{PANSS}_{-{ }_{\text {follow-up }}} \mathrm{PANSS}_{-} \mathrm{Pas}_{\text {ba }}\right.$ eline $\left.\left.) / \mathrm{PANSS}_{- \text {baseline }} ; \triangle \mathrm{PANSS}_{\text {negative } \%}\right)\right]$ positively correlated with baseline IL-27 $(\mathrm{Q}=0.785, \mathrm{P}=0.001)$, baseline IL-6 levels $(\mathrm{Q}=0.685$, $\mathrm{P}=0.007$ ), as they are presented on Table III and Figs. 1-4.

Following correlation analyses, association analyses were performed between PANSS subscales and each significantly correlated cytokine. As a result, based on the optimal regression equation indicated by the SPSS 'Curve Estimation' function, a linear association was observed between Follow-up PANSS $_{\text {negative }}$ was associated with baseline IL-27 levels $\left(\mathrm{R}^{2}=0.301, \mathrm{P}=0.042\right)$. Finally, $\triangle \mathrm{PANSS} \mathrm{negative}_{\mathrm{w}}$ was associated with baseline IL-6 levels $\left(\mathrm{R}^{2}=0.301, \mathrm{P}=0.042\right)$ and baseline IL-27 levels $\left(\mathrm{R}^{2}=0.446, \mathrm{P}=0.009\right)$ (Figs. 1-4). 
Table III. Statistically significant correlations between PANSS-negative, and IL-6 and IL-27 levels.

\section{Biomarkers}

\begin{tabular}{lcccr}
\cline { 2 - 3 } PANSS measurements & \multicolumn{2}{c}{ Serum IL-6 levels } & & Serum IL-27 levels \\
\cline { 2 - 4 } & Spearman's @ & P-value & & Spearman's @ \\
\hline Follow-up PANSS & 0.685 & 0.007 & 0.785 & 0.001 \\
PANSS $_{\text {negative\% }}$ & 0.685 & 0.007 & 0.785 & 0.001 \\
\hline
\end{tabular}

PANSS, positive and negative syndrome scale; IL, interleukin.

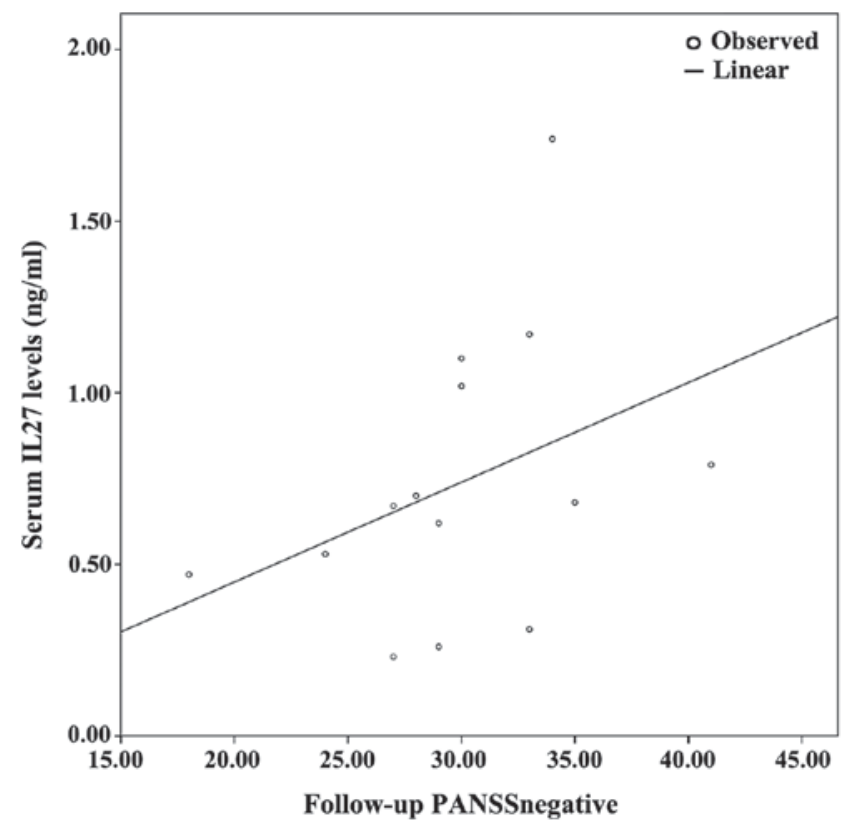

Figure 2. Combined graph depicting the scatterplot and linear regression line between serum IL-27 levels and follow-up PANSS-negative. IL-27, interleukin-27; PANSS, positive and negative syndrome scale.

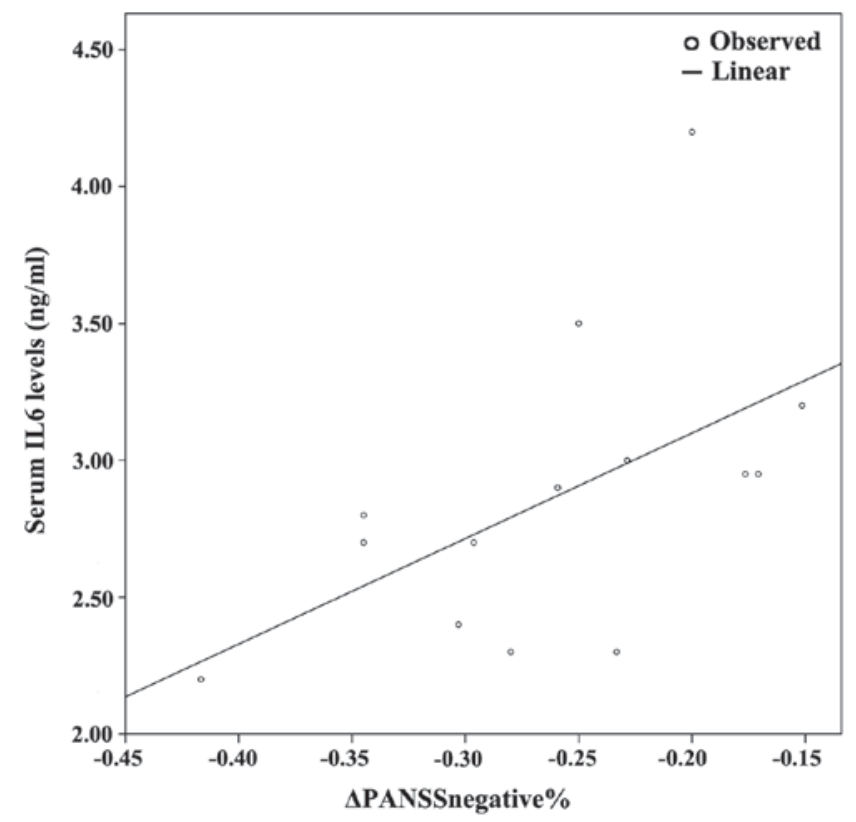

Figure 3. Combined graph depicting the scatterplot and linear regression line

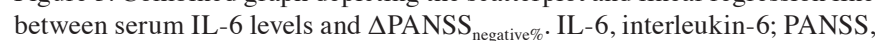
positive and negative syndrome scale.

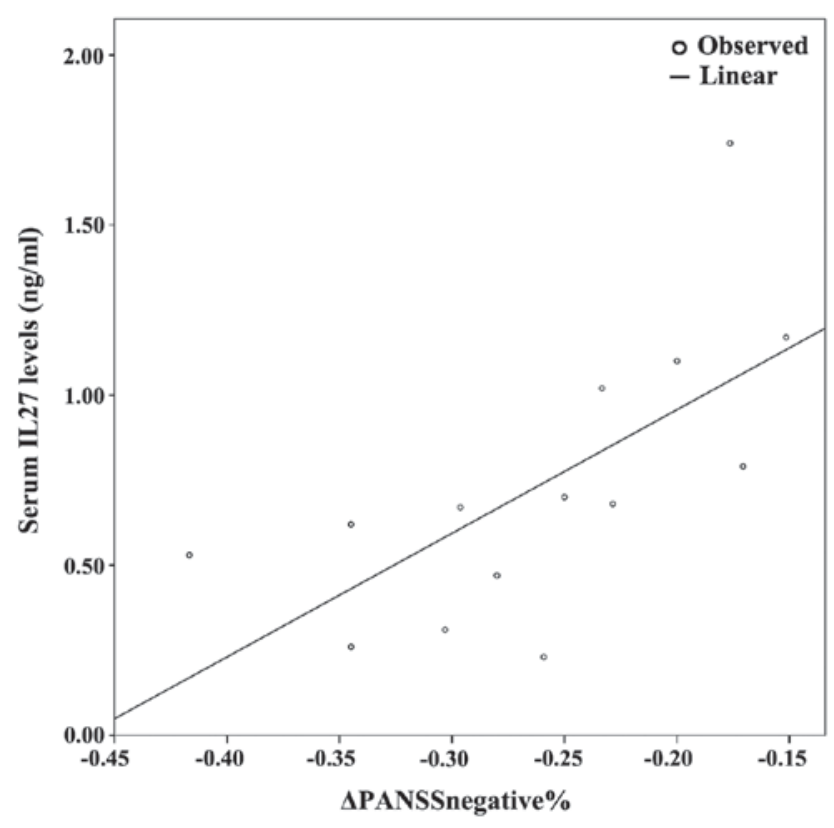

Figure 4. Combined graph depicting the scatterplot and linear regression line between serum IL-27 levels and $\triangle$ PANSS $_{\text {negative\% }}$. IL-27, interleukin-27; PANSS, positive and negative syndrome scale.

\section{Discussion}

In this study, we aimed to explore the clinical importance of serum biomarker levels i.e., cytokines and BDNF, in patients with FEP, regarding their response to treatment, while also adjusting for differences in baseline clinical characteristics as reflected on the PANSS subscales. Among the 15 cytokines measured, IL-6 and IL-27 were determined as the most important predictors of follow-up PANSS and percent change in PANSS negative subscales. While the initial positive correlation between baseline IL-6 and IL-27 with follow-up PANSS would imply that high IL-6 and IL-27 levels are associated with worse outcomes, the positive association with the percentage decrease in the PANSS negative scale would account for high IL-6, IL-27 levels being associated with a response to olanzapine treatment. On the other hand, no significant correlation was found between BDNF and PANSS (data not shown).

Disruptions in the Th1/Th2/Th17 cytokine immune response have been related to the pathogenesis of a number of disorders, including schizophrenia $(16,17)$. A recent meta-analysis provided clear evidence for the elevation of IL-1 $\beta$, sIL-2r, 
IL-6 and TNF- $\alpha$ levels in drug-naive FEP clearly suggesting a pro-inflammatory effect (18). In addition, Khandaker et al suggested that antipsychotic-naive FEP was associated with increased serum concentrations of pro-inflammatory cytokines, particularly IL-6, as well as TNF- $\alpha$, IL-1 $\beta$, INF- $\gamma$, and decreased serum concentrations of the anti-inflammatory cytokine IL-10, which were normalized following the remission of symptoms with antipsychotic treatment (19).

These changes in cytokine profiles have been associated with the onset of schizophrenia and seems that antipsychotic agents can modulate this profile. Moreover, an association between antipsychotic treatment and lower levels of IL-4, IL-6 and IL-27 in the FEP group has been reported (20). This report describes the distinct roles of IL-27, one as an immune response initiator, and the other as an immune response attenuator $(21,22)$. IL-27 can help differentiation and/or maintenance of Th1 cells, but can also suppress Th17 differentiation and IL-17 production (22). On the other hand, IL-27 may prevent excessive inflammation in autoimmune diseases (23). Furthermore, Borovcanin et al observed lower IL-27 levels in patients with FEP following treatment with an antipsychotic, which could indicate a predominance of a type-17 response (20).

IL-27, which has structural and functional similarities to IL-12, was identified by Pflanz et al 2002 (24). IL-27 promotes early Th1 differentiation through the upregulation of intercellular adhesion molecule 1 (ICAM-1) and T-bet, but suppresses Th2 and Th17 differentiation and the production of pro-inflammatory cytokines. One possible mechanism of its suppressive function may be through IL-10 production. In addition, IL-27 and TGF play a central role in generating IL-10-producing anti-inflammatory $\operatorname{Tr} 1$ cells (23).

IL-6 is chiefly produced by activated monocytes, and is often considered a component of the type- 2 immune response, even though it acts in conjunction with pro-inflammatory cytokines (25). It has been suggested that IL-6 may be involved in clinical symptomatology and clinical improvement in schizophrenia through immunological mechanisms. This is currently a debated hypothesis, with some studies reporting a positive correlation between IL-6 and positive, but also negative, PANSS scores $(26,27)$, while others have found no such association (28).

BDNF is a member of the neurotrophic family of growth factors that promotes the development, regeneration, sustaining and maintenance of neuron function in the central nervous system (29). The precursor protein, preproBDNF, is synthesized in the endoplasmic reticulum and then converted to proBDNF, which is proteolytically processed into mature BDNF. Yoshida et al suggested that proBDNF and mature BDNF affect CNS in an opposing manner, as only secreted mature BDNF was biologically active (30). Mature BDNF has an anti-inflammatory activity and thus it reduces the level of inflammation. The significant role of BDNF in the pathogenesis of the schizophrenic disorder could be reflected by the low serum BDNF levels to the onset of the disorder and the duration of untreated psychosis (31). Furthermore, the response of antipsychotics has also been linked with alterations in serum BDNF levels in patients with schizophrenia, suggesting a neurotrophic role for some second generation antipsychotics through their specific dopaminergic activity in the treatment of psychotic symptoms (32).
The lack of a correlation between BDNF and PANSS in this study, could be a direct cause of the assay used (BDNF ELISA kit; R\&D Systems), since it does not differentiate between proBDNF (precursor BDNF) and BDNF (mature BDNF), measuring them as total BDNF levels and not separately.

This study should be interpreted in lights of its limitations. First of all, the sample size of this study was small. Secondly, the serum levels of cytokines and BDNF were evaluated only twice, in a period of 8 weeks. On the contrary, this study consisted of exclusively drug-naive pure schizophrenic patients, a well-balanced sample of sex and age and monotherapy antipsychotic treatment.

The findings of this study suggest that drug-naive patients who exhibit high levels of IL-6 and IL-27 during the first episode of schizophrenia had a significant reduction in psychological symptoms and particularly in the negative symptoms after monotherapy with OLZ. Further longitudinal studies are warranted with larger sample sizes to validate these associations and to determine how these may serve as potential biomarkers of responsiveness and safety and to suggest novel immunomodulatory strategies for the treatment of schizophrenia.

\section{Acknowledgements}

The authors express their gratitude to the participants for their time and involvement in the study.

\section{Funding}

No funding was received.

\section{Availability of data and materials}

All data generated or analyzed during this study are included in this published article or are available from the corresponding author on reasonable request.

\section{Authors' contributions}

Authors EH, CP, NS and ER conceived and designed, and wrote the study. GDV, KT and NC undertook the statistical analysis. AT, AS and AD performed the analysis of cytokine levels. EL, DAS and NN performed the analysis of the BDNF levels. $\mathrm{CG}$ managed the literature search and wrote the first draft of the manuscript. All authors contributed to and have approved the final manuscript.

\section{Ethics approval and consent to participate}

This study acquired ethics approval by the University General Hospital 'ATTIKON'. All study participants supplied written informed consent. Participation in the study was voluntary and participants could withdraw at any point without detriment to their care. All participants had their capacity to consent tested by a clinical psychiatrist, while relatives or carers were present to ensure consent was properly informed.

\section{Patient consent for publication}

Not applicable. 


\section{Competing interests}

DAS is the Editor-in-Chief for the journal, but had no personal involvement in the reviewing process, or any influence in terms of adjudicating on the final decision, for this article. The other authors declare that they have no conflicts of interest.

\section{References}

1. van Os J and Kapur S: Schizophrenia. Lancet 374: 635-645, 2009.

2. Jenkins TA: Perinatal complications and schizophrenia: Involvement of the immune system. Front Neurosci 7: 110, 2013.

3. Benros ME, Nielsen PR, Nordentoft M, Eaton WW, Dalton SO and Mortensen PB: Autoimmune diseases and severe infections as risk factors for schizophrenia: A 30-year population-based register study. Am J Psychiatry 168: 1303-1310, 2011.

4. van Berckel BN, Bossong MG, Boellaard R, Kloet $R$, Schuitemaker A, Caspers E, Luurtsema G, Windhorst AD, Cahn W, Lammertsma AA, et al: Microglia activation in recentonset schizophrenia: A quantitative (R)-[11C]PK11195 positron emission tomography study. Biol Psychiatry 64: 820-822, 2008.

5. Doorduin J, de Vries EF, Willemsen AT, de Groot JC, Dierckx RA and Klein HC: Neuroinflammation in schizophrenia-related psychosis: A PET study. J Nucl Med 50: 1801-1807, 2009.

6. Potvin S, Stip E, Sepehry AA, Gendron A, Bah R and Kouassi E: Inflammatory cytokine alterations in schizophrenia: A systematic quantitative review. Biol Psychiatry 63: 801-808, 2008.

7. Miller BJ, Buckley P, Seabolt W, Mellor A and Kirkpatrick B: Meta-analysis of cytokine alterations in schizophrenia: Clinical status and antipsychotic effects. Biol Psychiatry 70: 663-671, 2011.

8. Lieberman JA, Stroup TS, McEvoy JP, Swartz MS, Rosenheck RA, Perkins DO, Keefe RS, Davis SM, Davis CE, Lebowitz BD, et al; Clinical antipsychotic trials of intervention effectiveness (CATIE) investigators: Effectiveness of antipsychotic drugs in patients with chronic schizophrenia. N Engl J Med 353: 1209-1223, 2005.

9. Drzyzga L, Obuchowicz E, Marcinowska A and Herman ZS: Cytokines in schizophrenia and the effects of antipsychotic drugs. Brain Behav Immun 20: 532-545, 2006.

10. Maes M, Bosmans E, Calabrese J, Smith R and Meltzer HY: Interleukin-2 and interleukin-6 in schizophrenia and mania: Effects of neuroleptics and mood stabilizers. J Psychiatr Res 29: 141-152, 1995

11. Müller N, Empl M, Riedel M, Schwarz M and Ackenheil M: Neuroleptic treatment increases soluble IL-2 receptors and decreases soluble IL-6 receptors in schizophrenia. Eur Arch Psychiatry Clin Neurosci 247: 308-313, 1997.

12. Meyer U, Schwarz MJ and Müller N: Inflammatory processes in schizophrenia: A promising neuroimmunological target for the treatment of negative/cognitive symptoms and beyond. Pharmacol Ther 132: 96-110, 2011.

13. American Psychiatric Association: Diagnostic and Statistical Manual of Mental Disorders. 4th edition. American Psychiatric Association, Washington, DC, 1994.

14. Kay SR, Fiszbein A and Opler LA: The positive and negative syndrome scale (PANSS) for schizophrenia. Schizophr Bull 13: 261-276, 1987.

15. Opler MGA, Yavorsky C and Daniel DG: Positive and Negative Syndrome Scale (PANSS) training: Challenges, solutions, and future directions. Innov Clin Neurosci 14: 77-81, 2017.

16. Müller N, Riedel M, Ackenheil M and Schwarz MJ: The role of immune function in schizophrenia: An overview. Eur Arch Psychiatry Clin Neurosci 249 (Suppl 4): 62-68, 1999.

17. Drexhage RC, Hoogenboezem TA, Cohen D, Versnel MA, Nolen WA, van Beveren NJ and Drexhage HA: An activated set point of T-cell and monocyte inflammatory networks in recent-onset schizophrenia patients involves both pro- and anti-inflammatory forces. Int J Neuropsychopharmacol 14: 746-755, 2011.
18. Upthegrove R, Manzanares-Teson N and Barnes NM: Cytokine function in medication-naive first episode psychosis: A systematic review and meta-analysis. Schizophr Res 155: 101-108, 2014.

19. Khandaker GM, Cousins L, Deakin J, Lennox BR, Yolken R and Jones PB: Inflammation and immunity in schizophrenia: Implications for pathophysiology and treatment. Lancet Psychiatry 2: 258-270, 2015.

20. Borovcanin M, Jovanovic I, Radosavljevic G, Djukic Dejanovic S, Stefanovic V, Arsenijevic N and Lukic ML: Antipsychotics can modulate the cytokine profile in schizophrenia: Attenuation of the type-2 inflammatory response. Schizophr Res 147: 103-109, 2013.

21. Gabay C and McInnes IB: The biological and clinical importance of the 'new generation' cytokines in rheumatic diseases. Arthritis Res Ther 11: 230, 2009.

22. Yoshida H, Nakaya $M$ and Miyazaki Y: Interleukin 27: A double-edged sword for offense and defense. J Leukoc Biol 86: 1295-1303, 2009.

23. Xu M, Mizoguchi I, Morishima N, Chiba Y, Mizuguchi J and Yoshimoto T: Regulation of antitumor immune responses by the IL-12 family cytokines, IL-12, IL-23 and IL-27. Clin Dev Immunol 2010: 2010, 2010.

24. Pflanz S, Timans JC, Cheung J, Rosales R, Kanzler H, Gilbert J, Hibbert L, Churakova T, Travis M, Vaisberg E, et al: IL-27, a heterodimeric cytokine composed of EBI3 and p28 protein, induces proliferation of naive $\mathrm{CD} 4^{+} \mathrm{T}$ cells. Immunity 16 : 779-790, 2002

25. Müller $N$ and Schwarz MJ: Immune system and schizophrenia. Curr Immunol Rev 6: 213-220, 2010.

26. Hori H, Yoshimura R, Yamada Y, Ikenouchi A, Mitoma M, Ida Y and Nakamura J: Effects of olanzapine on plasma levels of catecholamine metabolites, cytokines, and brainderived neurotrophic factor in schizophrenic patients. Int Clin Psychopharmacol 22: 21-27, 2007.

27. Pae CU, Yoon CH, Kim TS, Kim JJ, Park SH, Lee CU, Lee SJ, Lee $\mathrm{C}$ and Paik IH: Antipsychotic treatment may alter T-helper (TH)2 arm cytokines. Int Immunopharmacol 6: 666-671, 2006.

28. Zhang XY, Zhou DF, Zhang PY, Wu GY, Cao LY and Shen YC: Elevated interleukin-2, interleukin-6 and interleukin-8 serum levels in neuroleptic-free schizophrenia: Association with psychopathology. Schizophr Res 57: 247-258, 2002.

29. Maisonpierre PC, Belluscio L, Friedman B, Alderson RF, Wiegand SJ, Furth ME, Lindsay RM and Yancopoulos GD: NT-3, BDNF, and NGF in the developing rat nervous system: Parallel as well as reciprocal patterns of expression. Neuron 5: 501-509, 1990.

30. Yoshida T, Ishikawa M, Niitsu T, Nakazato M, Watanabe $H$, Shiraishi T, Shiina A, Hashimoto T, Kanahara N, Hasegawa T, et al: Decreased serum levels of mature brain-derived neurotrophic factor (BDNF), but not its precursor proBDNF, in patients with major depressive disorder. PLoS One 7: e42676, 2012.

31. Rizos EN, Papadopoulou A, Laskos E, Michalopoulou PG, Kastania A, Vasilopoulos D, Katsafouros K and Lykouras L: Reduced serum BDNF levels in patients with chronic schizophrenic disorder in relapse, who were treated with typical or atypical antipsychotics. World J Biol Psychiatry 11: 251-255, 2010.

32. Rizos EN, Michalopoulou PG, Siafakas N, Stefanis N, Douzenis A, Rontos I, Laskos E, Kastania A, Zoumpourlis V and Lykouras L: Association of serum brain-derived neurotrophic factor and duration of untreated psychosis in first-episode patients with schizophrenia. Neuropsychobiology 62: 87-90, 2010.

This work is licensed under a Creative Commons Attribution-NonCommercial-NoDerivatives 4.0 International (CC BY-NC-ND 4.0) License. 